\title{
Torsion free equiaffine connections on three-dimensional homogeneous spaces
}

\author{
Natalya P. Mozhey \\ Belarusian State University of Informatics and Radioelectronics \\ P. Brovki Street, 6, Minsk, 220013 Belarus
}

Received 22 February 2020, accepted 9 May 2020, published online 10 March 2021

\begin{abstract}
The aim of this paper is to describe equiaffine connections on three-dimensional homogeneous spaces. The affine connection is equiaffine if it admits a parallel volume form. Only the case of spaces not admitting connections with nonzero torsion is considered. For such homogeneous spaces, it is determined under what conditions the connection is equiaffine (locally equiaffine). In addition, equiaffine (locally equiaffine) connections and Ricci tensors are written out in explicit form. In this work we use the algebraic approach for description of connections, methods of the theory of Lie groups, Lie algebras and homogeneous spaces.
\end{abstract}

Keywords: Equiaffine connection, homogeneous space, transformation group, Lie algebra, torsion tensor, Ricci tensor.

Math. Subj. Class.: 53B05

\section{Introduction}

The aim of this paper is to describe equiaffine connections on three-dimensional homogeneous spaces. Only the case of spaces not admitting connections with nonzero torsion is considered. The case of affine connections is known (see [2]). The affine connection is equiaffine if it admits a parallel volume form (see [4]). For all such spaces, it is determined under what conditions the connection is equiaffine (locally equiaffine). In addition, equiaffine (locally equiaffine) connections and Ricci tensors are written out in explicit form.

Let $(\bar{G}, M)$ be a three-dimensional homogeneous space, where $\bar{G}$ is a Lie group acts transitively on the manifold $M$. We fix an arbitrary point $o \in M$ and denote by $G=\bar{G}_{o}$ the stationary subgroup of $o$. Then we can correspond the pair $(\overline{\mathfrak{g}}, \mathfrak{g})$ of Lie algebras to $(\bar{G}, G)$, where $\overline{\mathfrak{g}}$ is the Lie algebra of $\bar{G}$ and $\mathfrak{g}$ is the subalgebra of $\overline{\mathfrak{g}}$ corresponding to the subgroup

E-mail address: mozheynatalya@mail.ru (Natalya P. Mozhey) 
$G$. The pair $(\overline{\mathfrak{g}}, \mathfrak{g})$ is said to be isotropy-faithful if its isotropic representation is injective. The classification all three-dimensional isotropically-faithful pairs $(\overline{\mathfrak{g}}, \mathfrak{g})$ with torsion-free connections only, is described in [2]. Let $\mathfrak{m}=\overline{\mathfrak{g}} / \mathfrak{g}$. Invariant affine connections on $(\bar{G}, M)$ are in one-to-one correspondence [3] with linear mappings $\Lambda: \overline{\mathfrak{g}} \rightarrow \mathfrak{g l}(\mathfrak{m})$ such that $\left.\Lambda\right|_{\mathfrak{g}}=$ $\lambda$ and $\Lambda$ is $\mathfrak{g}$-invariant. We call this mappings (invariant) affine connections on the pair $(\overline{\mathfrak{g}}, \mathfrak{g})$. If there exists at least one invariant connection on $(\overline{\mathfrak{g}}, \mathfrak{g})$ then this pair is isotropyfaithful [1]. The curvature and torsion tensors of the invariant affine connection $\Lambda$ are given by the following formulas: $R: \mathfrak{m} \wedge \mathfrak{m} \rightarrow \mathfrak{g l}(\mathfrak{m}),\left(x_{1}+\mathfrak{g}\right) \wedge\left(x_{2}+\mathfrak{g}\right) \mapsto\left[\Lambda\left(x_{1}\right), \Lambda\left(x_{2}\right)\right]-$ $\Lambda\left(\left[x_{1}, x_{2}\right]\right) ; T: \mathfrak{m} \wedge \mathfrak{m} \rightarrow \mathfrak{m},\left(x_{1}+\mathfrak{g}\right) \wedge\left(x_{2}+\mathfrak{g}\right) \mapsto \Lambda\left(x_{1}\right)\left(x_{2}+\mathfrak{g}\right)-\Lambda\left(x_{2}\right)\left(x_{1}+\mathfrak{g}\right)-\left[x_{1}, x_{2}\right]_{\mathfrak{m}}$. The connection $\Lambda$ is torsion-free (or without torsion) if $T=0$. In this case we have: $R(x, y) z+R(y, z) x+R(z, x) y=0$ for all $x, y, z \in \mathfrak{m}$ (the first Bianchi identity).

We define the Ricci tensor: $\operatorname{Ric}(y, z)=\operatorname{tr}\{x \mapsto R(x, y) z\}$. An affine connection $\Lambda$ with zero torsion has symmetric Ricci tensor if and only if it is locally equiaffine [4]. Really, $\operatorname{Ric}(y, z)-\operatorname{Ric}(z, y)=\operatorname{tr}\{x \mapsto R(x, y) z-R(x, z) y\}$. From the first Bianchi identity we obtain $\operatorname{Ric}(y, z)-\operatorname{Ric}(z, y)=\operatorname{tr}\{x \mapsto-R(y, z) x\}=-\operatorname{tr} R(y, z)$. Then $\operatorname{Ric}(y, z) \operatorname{Ric}(z, y)=$ $-\operatorname{tr}(\Lambda(y) \Lambda(z)-\Lambda(z) \Lambda(y))+\operatorname{tr} \Lambda([y, z])=\operatorname{tr} \Lambda([y, z])$. Hence Ric is symmetric if and only if $\operatorname{tr} \Lambda([y, z])=0$ for all $y, z \in \overline{\mathfrak{g}}$. We say that the affine connection $\Lambda$ is locally equiaffine if $\operatorname{tr} \Lambda([x, y])=0$ for all $x, y \in \overline{\mathfrak{g}}$ (i.e. $\Lambda([\overline{\mathfrak{g}}, \overline{\mathfrak{g}}]) \subset \mathfrak{s l}(\mathfrak{m}))$. By equiaffine connection we mean the (torsion-free) affine connection $\Lambda$ such that $\operatorname{tr} \Lambda(x)=0$ for all $x \in \overline{\mathfrak{g}}$. In this case, it is obvious $\lambda(\mathfrak{g}) \subset \mathfrak{s l}(\mathfrak{m})$.

We define $(\overline{\mathfrak{g}}, \mathfrak{g})$ by the commutation table of the Lie algebra $\overline{\mathfrak{g}}$. Here by $\left\{e_{1}, \ldots, e_{n}\right\}$ we denote a basis of $\overline{\mathfrak{g}}(n=\operatorname{dim} \overline{\mathfrak{g}})$. We assume that the Lie algebra $\mathfrak{g}$ is generated by $e_{1}, \ldots, e_{n-3}$. Let $\left\{u_{1}=e_{n-2}, u_{2}=e_{n-1}, u_{3}=e_{n}\right\}$ be a basis of $\mathfrak{m}$. We describe affine connection by $\Lambda\left(u_{1}\right), \Lambda\left(u_{2}\right), \Lambda\left(u_{3}\right)$, curvature tensor $R$ by $R\left(u_{1}, u_{2}\right), R\left(u_{1}, u_{3}\right), R\left(u_{2}, u_{3}\right)$ and torsion tensor $T$ by $T\left(u_{1}, u_{2}\right), T\left(u_{1}, u_{3}\right), T\left(u_{2}, u_{3}\right)$. We say that the affine connection is trivial if $\Lambda\left(u_{1}\right)=\Lambda\left(u_{2}\right)=\Lambda\left(u_{3}\right)=0$. To refer to the pair we use the notation d.n.m, where $d$ is the dimension of the subalgebra, $n$ is the number of the subalgebra of $\mathfrak{g l}(3, \mathbb{R})$, $m$ is the number of $(\overline{\mathfrak{g}}, \mathfrak{g})$ in [2].

The description of (torsion-free) equiaffine connections on three-dimensional homogeneous spaces can be divided into the following parts:

- classification of pairs that allow nontrivial locally equiaffine connections (the curvature tensor is only zero in Theorem 2.1; the curvature tensor is not only zero in Theorem 2.2);

- classification of pairs with only trivial locally equiaffine connections (the curvature tensor is zero in Theorem 3.1; the curvature tensor is not zero in Theorem 3.2).

The information about equiaffine (locally equiaffine) connections and Ricci tensors is contained in the proof of the Theorems 2.1, 2.2, 3.1 and 3.2.

\section{Pairs of Lie algebras, admitting nontrivial locally equiaffine connec- tions}

\subsection{The curvature tensor is only zero}

Theorem 2.1. I. If the pair $(\overline{\mathfrak{g}}, \mathfrak{g})$ allows nontrivial equiaffine connections, the curvature and torsion tensors are only zero, then $(\overline{\mathfrak{g}}, \mathfrak{g})$ is equivalent to one and only one of the following pairs: 
$-\overline{\mathfrak{g}}$ is nonsolvable:

\begin{tabular}{c|ccccccc}
4.21 .11$. & $e_{1}$ & $e_{2}$ & $e_{3}$ & $e_{4}$ & $u_{1}$ & $u_{2}$ & $u_{3}$ \\
\hline$e_{1}$ & 0 & $e_{2}$ & $-\mu e_{3}$ & $(1-\mu) e_{4}$ & $u_{1}$ & 0 & $\mu u_{3}$ \\
$e_{2}$ & $-e_{2}$ & 0 & $e_{4}$ & 0 & 0 & $e_{2}+u_{1}$ & 0 \\
$e_{3}$ & $\mu e_{3}$ & $-e_{4}$ & 0 & 0 & 0 & $-2 e_{3}$ & $u_{2}$ \\
$e_{4}$ & $(\mu-1) e_{4}$ & 0 & 0 & 0 & 0 & $-e_{4}$ & $e_{2}+u_{1}$ \\
$u_{1}$ & $-u_{1}$ & 0 & 0 & 0 & 0 & 0 & 0 \\
$u_{2}$ & 0 & $-e_{2}-u_{1}$ & $2 e_{3}$ & $e_{4}$ & 0 & 0 & $-2 u_{3}$ \\
$u_{3}$ & $-\mu u_{3}$ & 0 & $-u_{2}$ & $-e_{2}-u_{1}$ & 0 & $2 u_{3}$ & 0
\end{tabular}

$-\overline{\mathfrak{g}}$ is solvable:

\begin{tabular}{c|ccccc}
1.2 .1 & $e_{1}$ & $u_{1}$ & $u_{2}$ & $u_{3}$ & \\
\cline { 1 - 4 }$e_{1}$ & 0 & $u_{1}$ & $\lambda u_{2}$ & $\mu u_{3}$ & \\
$u_{1}$ & $-u_{1}$ & 0 & 0 & 0 & $\lambda=-2 / 3$ \\
$u_{2}$ & $-\lambda u_{2}$ & 0 & 0 & 0 & \\
$u_{3}$ & $-\mu u_{3}$ & 0 & 0 & 0 &
\end{tabular}

\begin{tabular}{c|cccccc}
2.9 .1$. & $e_{1}$ & $e_{2}$ & $u_{1}$ & $u_{2}$ & $u_{3}$ & \\
\cline { 1 - 5 }$e_{1}$ & 0 & $(1-\mu) e_{2}$ & $u_{1}$ & $\lambda u_{2}$ & $\mu u_{3}$ & \\
$e_{2}$ & $(\mu-1) e_{2}$ & 0 & 0 & 0 & $u_{1}$ & $\lambda=-3 / 2, \mu=1 / 2 ;$ \\
$u_{1}$ & $-u_{1}$ & 0 & 0 & 0 & 0 & $\lambda=-2 / 3, \mu=-1 / 3$ \\
$u_{2}$ & $-\lambda u_{2}$ & 0 & 0 & 0 & 0 & $\lambda=1 / 2, \mu=-3 / 2$ \\
$u_{3}$ & $-\mu u_{3}$ & $-u_{1}$ & 0 & 0 & 0 &
\end{tabular}

\begin{tabular}{c|cccccc}
$3.20 .25, \mu \leq 0$ & $e_{1}$ & $e_{2}$ & $e_{3}$ & $u_{1}$ & $u_{2}$ & $u_{3}$ \\
\hline$e_{1}$ & 0 & $(1-2 \mu) e_{2}$ & $(1-\mu) e_{3}$ & $u_{1}$ & $2 \mu u_{2}$ & $\mu u_{3}$ \\
$e_{2}$ & $(2 \mu-1) e_{2}$ & 0 & 0 & 0 & $u_{1}$ & $e_{3}$ \\
$e_{3}$ & $(\mu-1) e_{3}$ & 0 & 0 & 0 & 0 & $u_{1}$ \\
$u_{1}$ & $-u_{1}$ & 0 & 0 & 0 & 0 & 0 \\
$u_{2}$ & $-2 \mu u_{2}$ & $-u_{1}$ & 0 & 0 & 0 & 0 \\
$u_{3}$ & $-\mu u_{3}$ & $-e_{3}$ & $-u_{1}$ & 0 & 0 & 0
\end{tabular}

The Ricci tensors are zero.

II. Any pair $(\overline{\mathfrak{g}}, \mathfrak{g})$, allows nontrivial affine connections, the curvature and torsion tensors are only zero (i.e. if $\overline{\mathfrak{g}}$ is nonsolvable then d.n.m $=6.3 .2,5.9 .2,4.19 .2,4.21 .11$ $(\mu \neq 0,1,1 / 2), 3.6 .2,3.12 .2,3.13 .6(\mu \neq 0,1,-1,1 / 2), 3.28 .2,2.8 .7(\lambda \neq 0,1,-1,1 / 2)$, if $\overline{\mathfrak{g}}$ is solvable then d.n. $m=5.10 .1(\lambda=1 / 2, \mu=0), 4.8 .1(\lambda=0, \mu=1 / 2), 4.11 .1(\mu=0, \lambda=1 / 2)$, 4.11.5, 3.7.1 ( $\lambda=1 / 2), 3.8 .1$ ( $\lambda \nmid \mu=1 / 2, \mu=0,1 / 2), 3.14 .1(\mu \neq 0,2), 3.19 .17,3.20 .1(\lambda=1 / 2$ $(\mu \neq 0,1 / 2) ; \mu=1 / 2(\lambda \neq 0,1 / 2)), 3.20 .25(\mu \neq 0), 3.20 .26(\lambda \neq 1 / 3,1 / 4), 3.23 .1(\lambda=3 / 4)$, 3.29.1 $(\mu=1 / 2), 2.1 .1(\lambda=1 / 2), 2.8 .1(\lambda=1 / 2), 2.9 .1(\lambda=1 / 2(\mu \neq 0,-1 / 2,1 / 4,1 / 2)$; $\lambda=2 \mu(\mu \neq 0,1 / 4,1 / 3,1) ; \mu=1 / 2(\lambda \neq 1 / 2,0,1,3 / 2)), 2.19 .1(\lambda=1 / 2), 2.19 .5,2.21 .1$ $(\lambda=3 / 4), 1.2 .1(\mu=2 \lambda(\lambda \neq 1 / 3,1 / 4) ; \mu=\lambda / 2(\lambda \neq-2) ; \lambda=1 / 2(\mu \neq 1 / 2)), 1.7 .1(\lambda=1 / 2)$, see [2]), admits locally equiaffine connections.

Remark. In the cases 5.10.1 $(\lambda=1 / 2, \mu=0), 3.8 .1(\lambda=0, \mu=1 / 2), 3.20 .1(\lambda=1 / 2$ $(\mu \neq 0,1 / 2), \mu=1 / 2(\lambda \neq 0,1 / 2)), 3.23 .1(\lambda=3 / 4), 3.29 .1(\mu=1 / 2), 2.9 .1(\mu=1 / 2$ $(\lambda \neq 1 / 2,0,1,3 / 2)), 2.19 .1(\lambda=1 / 2)$ the connection is trivial after basis replacement. 
Proof. For the subalgebras $\mathfrak{g}$ of $\mathfrak{g l}(3, \mathbb{R})$ in [2] we find isotropy-faithful pairs $(\overline{\mathfrak{g}}, \mathfrak{g})$ and choose pairs, allows nontrivial affine, equiaffine (locally equiaffine) connections, such that the curvature and torsion tensors are zero for all connections.

Let $\overline{\mathfrak{g}}$ is nonsolvable, for example, the pair $(\overline{\mathfrak{g}}, \mathfrak{g})$ has the form 6.3 .2 and

$$
\Lambda\left(u_{1}\right)=\left(\begin{array}{lll}
p_{1,1} & p_{1,2} & p_{1,3} \\
p_{2,1} & p_{2,2} & p_{2,3} \\
p_{3,1} & p_{3,2} & p_{3,3}
\end{array}\right), \Lambda\left(u_{2}\right)=\left(\begin{array}{lll}
q_{1,1} & q_{1,2} & q_{1,3} \\
q_{2,1} & q_{2,2} & q_{2,3} \\
q_{3,1} & q_{3,2} & q_{3,3}
\end{array}\right), \quad \Lambda\left(u_{3}\right)=\left(\begin{array}{lll}
r_{1,1} & r_{1,2} & r_{1,3} \\
r_{2,1} & r_{2,2} & r_{2,3} \\
r_{3,1} & r_{3,2} & r_{3,3}
\end{array}\right)
$$

$p_{i, j}, q_{i, j}, r_{i, j} \in \mathbb{R}(i, j=1,2,3) .\left.\Lambda\right|_{\mathfrak{g}}$ is the isotropic representation of $\mathfrak{g}, \Lambda$ is $\mathfrak{g}$-invariant $\Rightarrow\left[\Lambda\left(e_{2}\right), \Lambda\left(u_{1}\right)\right]=0, p_{3,1}=p_{3,2}=p_{1,2}=0, p_{3,3}=p_{2,2} \cdot\left[\Lambda\left(e_{1}\right), \Lambda\left(u_{1}\right)\right]=\Lambda\left(\left[e_{1}, u_{1}\right]\right) \Rightarrow$ $p_{1,3}=p_{2,1}=p_{2,3}=0$. $\left[\Lambda\left(e_{5}\right), \Lambda\left(u_{1}\right)\right]=\Lambda\left(\left[e_{5}, u_{1}\right]\right) \Rightarrow p_{2,2}=p_{1,1}$. If $\left[\Lambda\left(e_{2}\right), \Lambda\left(u_{2}\right)\right]=0$ then $q_{3,1}=q_{3,2}=q_{1,2}=0, q_{3,3}=q_{2,2} \cdot\left[\Lambda\left(e_{1}\right), \Lambda\left(u_{2}\right)\right]=\Lambda\left(u_{2}\right), q_{1,1}=q_{2,2}=q_{2,3}=0$. $\left[\Lambda\left(e_{3}\right), \Lambda\left(u_{2}\right)\right]=\Lambda\left(u_{3}\right), r_{1,1}=r_{1,3}=r_{2,1}=r_{2,2}=r_{2,3}=r_{3,2}=r_{3,3}=0, r_{3,1}=q_{2,1}, r_{1,2}=$ $-q_{1,3}$. If $\left[\Lambda\left(e_{4}\right), \Lambda\left(u_{2}\right)\right]=\Lambda\left(u_{2}\right)$ then $r_{1,2}=0$. $\left[\Lambda\left(e_{5}\right), \Lambda\left(u_{2}\right)\right]=\Lambda\left(u_{1}\right)+\Lambda\left(e_{1}\right)+3 \Lambda\left(e_{4}\right)$, $p_{1,1}=r_{3,1}=-2, \operatorname{tr} \Lambda\left(3 e_{4}+u_{1}\right)=0 \Rightarrow \operatorname{tr} \Lambda([x, y])=0$ for all $x, y \in \overline{\mathfrak{g}}$, the connection is locally equiaffine and has the form, presented in the table, the curvature and torsion tensors are zero. In this case Ricci tensor is equal to zero too. We have $\operatorname{tr} \Lambda\left(e_{4}\right) \neq 0 \Rightarrow$ the connection is not equiaffine. In the cases 3.13.6 $(\mu=-1)$ and 2.8.7 $(\lambda=-1)$ the connection is equiaffine, but admitting nonzero torsion tensor.

Similarly we obtain the results in the other cases:

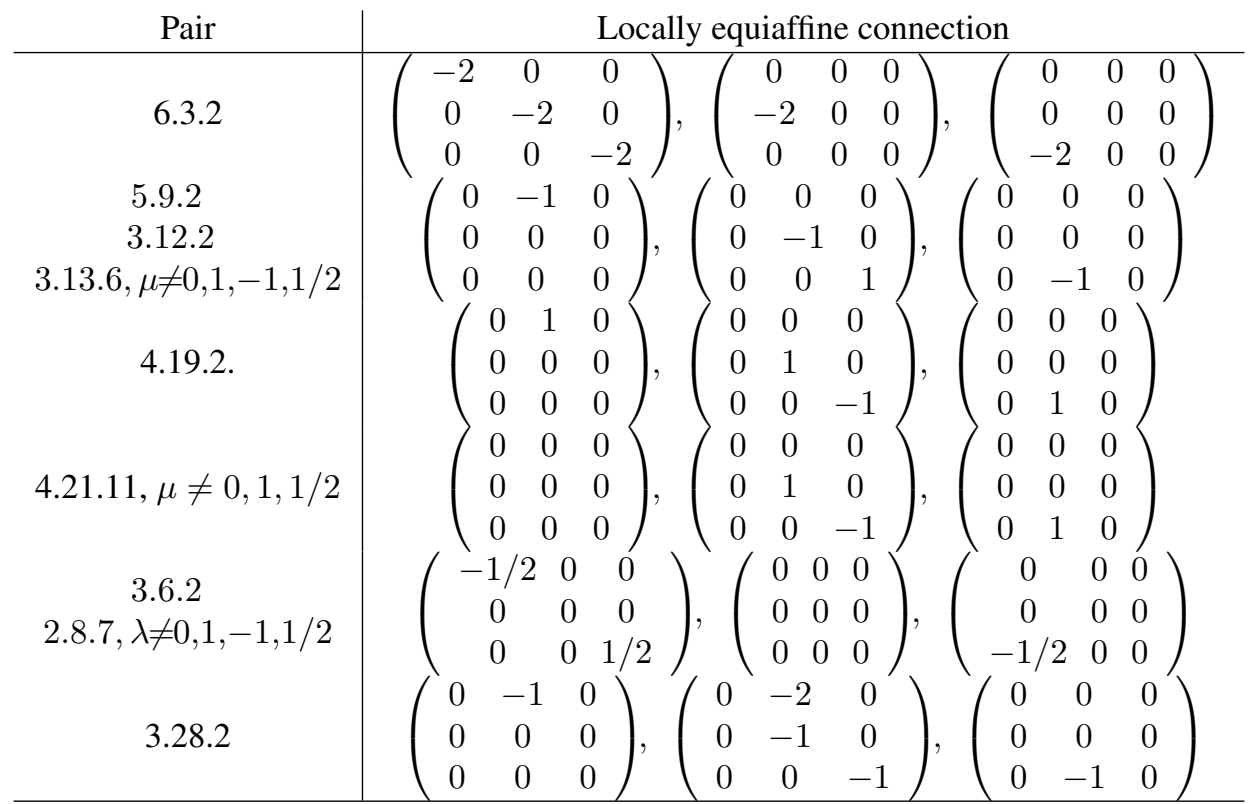

The connection is equiaffine only in the case $4.21 .11, \mu=-1$.

Let $\overline{\mathfrak{g}}$ is solvable, for example, $(\overline{\mathfrak{g}}, \mathfrak{g})$ is $5.10 .1(\lambda=1 / 2, \mu=0)$ then $\left.\Lambda\right|_{\mathfrak{g}}$ is the isotropic representation of $\mathfrak{g}$. $\Lambda$ is $\mathfrak{g}$-invariant $\Rightarrow \operatorname{tr} \Lambda([x, y])=0$ for all $x, y \in \overline{\mathfrak{g}}$ and locally equiaffine connection there exist and has the form, presented in the table, the Ricci tensor, curvature and torsion tensors are equal to zero. In this case $\operatorname{tr} \Lambda\left(e_{1}\right) \neq 0$ and the connection is not equiaffine. Similarly we obtain the results in the other cases: 


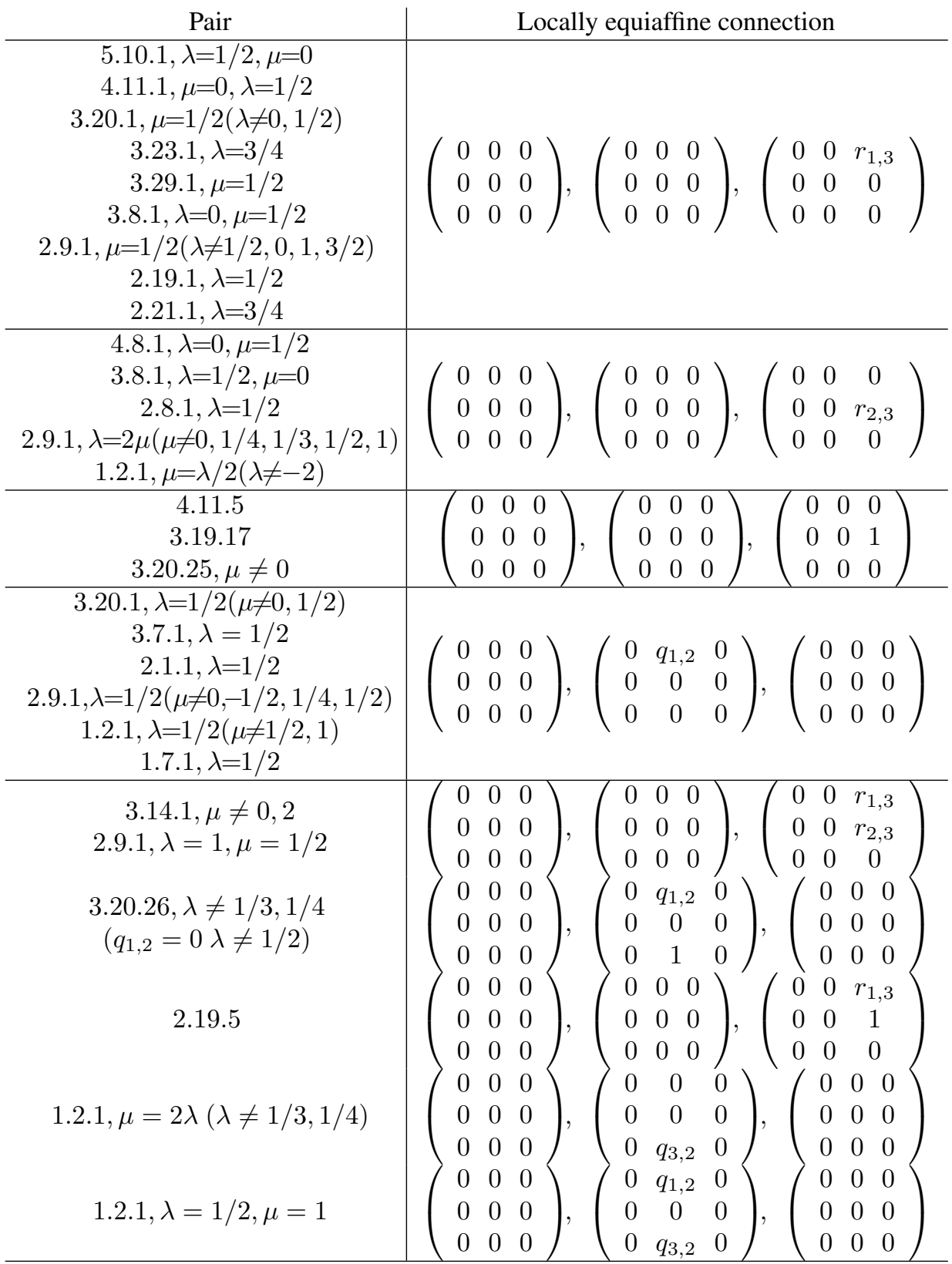

We have equiaffine connections only in the cases $2.9 .1, \lambda=-3 / 2, \mu=1 / 2 ; 1.2 .1, \lambda=$ $-2 / 3, \mu=-1 / 3$ and $2.9 .1, \lambda=-2 / 3, \mu=-1 / 3 ; 3.20 .25, \mu=-1 / 3 ; 2.9 .1, \lambda=1 / 2, \mu=-3 / 2$ (respectively).

The Ricci tensors $\operatorname{Ric}(y, z)=\operatorname{tr}\{x \mapsto R(x, y) z\}$ are equal to zero. 


\subsection{The curvature tensor is not only zero}

Theorem 2.2. I. There are no pairs $(\overline{\mathfrak{g}}, \mathfrak{g})$, admitting nontrivial equiaffine connections with nonzero curvature tensor and only zero torsion tensor.

II. Any pair $(\overline{\mathfrak{g}}, \mathfrak{g})$, allows nontrivial affine connections, the curvature tensor is not only zero, the torsion tensor is only zero (i.e. if $\overline{\mathfrak{g}}$ is nonsolvable then d.n.m $m=4.21 .11(\mu=1 / 2)$, 3.13.6 $(\mu=1 / 2)$, 2.8.7 $(\lambda=1 / 2)$, if $\overline{\mathfrak{g}}$ is solvable then d.n. $m=3.13 .2(\mu=1 / 2,1 / 4), 3.20 .4$ $(\lambda=1 / 4), 3.20 .26(\lambda=1 / 4), 3.20 .27,2.9 .1 \quad(\lambda=1 / 2, \mu=1 / 4), 2.9 .3(\mu=1 / 4), 1.2 .1 \quad(\lambda=1 / 4$, $\mu=1 / 2)$, see [2]), admits locally equiaffine connections. The Ricci tensors are zero.

Proof. Just as earlier, in case, for example, $3.13 .2(\mu=1 / 2,1 / 4)$ we have $\operatorname{tr} \Lambda([x, y])=0$ for all $x, y \in \overline{\mathfrak{g}}$, the torsion tensor and Ricci tensor are zero and locally equiaffine connection has the form, presented in the table. The connection is equiaffine if $\operatorname{tr} \Lambda\left(e_{1}\right)=0 \Rightarrow$ $\mu=2$, but in this case $0<\mu<1 \Rightarrow$ the pair does not allow equiaffine connections. In the case 3.20.4 the connection is equiaffine if $\lambda=2$, but $\lambda<1 / 3$. Similarly we obtain the results in other cases:

$-\overline{\mathfrak{g}}$ is solvable:

\begin{tabular}{|c|c|c|c|c|c|c|c|c|c|c|c|c|}
\hline Pair & \multicolumn{12}{|c|}{ Locally equiaffine connection } \\
\hline \multirow{3}{*}{$3.13 .2, \mu=1 / 4$} & & 0 & & 0 & 0 & 0 & 0 & & 0 & 0 & 0 & \\
\hline & & & 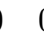 & 0 & 0 & 0 & 0 & & & 0 & $r_{2,3}$ & \\
\hline & & & & 0 & 0 & 0 & 0 & & 0 & 0 & 0 & \\
\hline \multirow{3}{*}{$\begin{array}{l}3.13 .2, \mu=1 / 2 \\
3.20 .4, \lambda=1 / 4\end{array}$} & & & 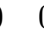 & & 0 & 0 & 0 & & 0 & 0 & $r_{1,3}$ & \\
\hline & & & ( & 0 & 0 & 0 & 0 & & 0 & 0 & 0 & \\
\hline & & & & 0 & 0 & 0 & 0 & & 0 & 0 & 0 & \\
\hline \multirow{3}{*}{$3.20 .26, \lambda=1 / 4$} & & & & & 0 & 0 & 0 & & 0 & 0 & $r_{1,3}$ & \\
\hline & & & 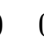 & 0 & 0 & 0 & 0 & & 0 & 0 & 0 & \\
\hline & & & & 0 & 0 & 1 & 0 & & 0 & 0 & 0 & \\
\hline \multirow{3}{*}{3.20 .27} & & & 0 & 0 & & $\begin{array}{ll}0 & 0\end{array}$ & & & & & 0 & \\
\hline & & 0 & 0 & 0 & & $0 \quad 0$ & 0 & & & & 0 & \\
\hline & & 0 & 0 & 0 & & $0 \quad 1$ & 0 & & & & & \\
\hline \multirow{4}{*}{$\begin{array}{c}2.9 .1, \lambda=1 / 2, \mu=1 / 4 \\
2.9 .3, \mu=1 / 4\end{array}$} & & 0 & 0 & & 0 & $q_{1,2}$ & 0 & & & & & \\
\hline & & 0 & 0 & , & 0 & 0 & 0 & & & & & 2,3 \\
\hline & & 0 & 0 & & 0 & 0 & 0 & & & & & 0 \\
\hline & ( & 0 & 0 & & 0 & 0 & 0 & & & & & 1,3 \\
\hline \multirow{2}{*}{$1.2 .1, \lambda=1 / 4, \mu=1 / 2$} & ( & 0 & 0 & & 0 & 0 & 0 & & & & & 0 \\
\hline & ( & 0 & 0 & & 0 & $q_{3,2}$ & 0 & & & & & 0 \\
\hline
\end{tabular}

$-\overline{\mathfrak{g}}$ is nonsolvable:

\begin{tabular}{c|c} 
Pair & $\begin{array}{c}\text { Locally equiaffine connection } \\
\text { 4.21.11, } \mu=1 / 2 \\
3.13 .6, \mu=1 / 2\end{array} \quad\left(\begin{array}{ccc}0 & 0 & 0 \\
0 & 0 & 0 \\
0 & 0 & 0\end{array}\right),\left(\begin{array}{ccc}0 & 0 & 0 \\
0 & 1 & 0 \\
0 & 0 & -1 \\
0 & 0 & 0 \\
0 & 0 & 0\end{array}\right),\left(\begin{array}{ccc}0 & 0 & r_{1,3} \\
0 & 0 & 0 \\
0 & 0 & 0 \\
0 & -1 & 0 \\
0 & 0 & r_{1,3} \\
0 & 0 & 0 \\
0 & 0 & 1 \\
0 & -1 & 0 \\
0 & 0 & 0 \\
0 & 0 & r_{2,3} \\
-1 / 2 & 0 & 0 \\
0 & 0 & 0 \\
0 & 0 & 1 / 2\end{array}\right),\left(\begin{array}{ccc}0 & 0 & 0 \\
0 & 0 & 0 \\
0 & 0 & 0\end{array}\right),\left(\begin{array}{ccc}0 & 0\end{array}\right)$ \\
\hline
\end{tabular}

In these cases, equiaffine connections does not exist.

The Ricci tensors are equal to zero. 


\section{Pairs of Lie algebras with only trivial locally equiaffine connections}

\subsection{The curvature tensor is only zero}

Theorem 3.1. I. If the pair $(\overline{\mathfrak{g}}, \mathfrak{g})$ admits only trivial equiaffine connection, the curvature and torsion tensors are zero, then $(\overline{\mathfrak{g}}, \mathfrak{g})$ is equivalent to one of the pairs:

$-\overline{\mathfrak{g}}$ is nonsolvable: $8.1 .1(\mathfrak{s l}(3, \mathbb{R}))$;

\begin{tabular}{c|ccccccccc}
6.2 .1 & $e_{1}$ & $e_{2}$ & $e_{3}$ & $e_{4}$ & $e_{5}$ & $e_{6}$ & $u_{1}$ & $u_{2}$ & $u_{3}$ \\
\hline$e_{1}$ & 0 & 0 & 0 & $(\lambda-1) e_{4}$ & 0 & $(\lambda-1) e_{6}$ & $\lambda u_{1}$ & $\lambda u_{2}$ & $u_{3}$ \\
$e_{2}$ & 0 & 0 & $2 e_{3}$ & $e_{4}$ & $-2 e_{5}$ & $-e_{6}$ & $u_{1}$ & $-u_{2}$ & 0 \\
$e_{3}$ & 0 & $-2 e_{3}$ & 0 & 0 & $e_{2}$ & $e_{4}$ & 0 & $u_{1}$ & 0 \\
$e_{4}$ & $(1-\lambda) e_{4}$ & $-e_{4}$ & 0 & 0 & $-e_{6}$ & 0 & 0 & 0 & $u_{1}$ \\
$e_{5}$ & 0 & $2 e_{5}$ & $-e_{2}$ & $e_{6}$ & 0 & 0 & $u_{2}$ & 0 & 0 \\
$e_{6}$ & $(1-\lambda) e_{6}$ & $e_{6}$ & $-e_{4}$ & 0 & 0 & 0 & 0 & 0 & $u_{2}$ \\
$u_{1}$ & $-\lambda u_{1}$ & $-u_{1}$ & 0 & 0 & $-u_{2}$ & 0 & 0 & 0 & 0 \\
$u_{2}$ & $-\lambda u_{2}$ & $u_{2}$ & $-u_{1}$ & 0 & 0 & 0 & 0 & 0 & 0 \\
$u_{3}$ & $-u_{3}$ & 0 & 0 & $-u_{1}$ & 0 & $-u_{2}$ & 0 & 0 & 0
\end{tabular}

\begin{tabular}{c|ccccccccc}
6.4 .1 & $e_{1}$ & $e_{2}$ & $e_{3}$ & $e_{4}$ & $e_{5}$ & $e_{6}$ & $u_{1}$ & $u_{2}$ & $u_{3}$ \\
\hline$e_{1}$ & 0 & 0 & 0 & 0 & $(1-\lambda) e_{5}$ & $(1-\lambda) e_{6}$ & $u_{1}$ & $\lambda u_{2}$ & $\lambda u_{3}$ \\
$e_{2}$ & 0 & 0 & $2 e_{3}$ & $-2 e_{4}$ & $-e_{5}$ & $e_{6}$ & 0 & $u_{2}$ & $-u_{3}$ \\
$e_{3}$ & 0 & $-2 e_{3}$ & 0 & $e_{2}$ & $-e_{6}$ & 0 & 0 & 0 & $u_{2}$ \\
$e_{4}$ & 0 & $2 e_{4}$ & $-e_{2}$ & 0 & 0 & $-e_{5}$ & 0 & $u_{3}$ & 0 \\
$e_{5}$ & $(\lambda-1) e_{5}$ & $e_{5}$ & $e_{6}$ & 0 & 0 & 0 & 0 & $u_{1}$ & 0
\end{tabular},$\lambda=-1 / 2 ;$

\begin{tabular}{c|ccccccc}
4.2 .1$. & $e_{1}$ & $e_{2}$ & $e_{3}$ & $e_{4}$ & $u_{1}$ & $u_{2}$ & $u_{3}$ \\
\hline$e_{1}$ & 0 & 0 & 0 & 0 & $\lambda u_{1}$ & $\lambda u_{2}$ & $u_{3}$ \\
$e_{2}$ & 0 & 0 & $2 e_{3}$ & $-2 e_{4}$ & $u_{1}$ & $-u_{2}$ & 0 \\
$e_{3}$ & 0 & $-2 e_{3}$ & 0 & $e_{2}$ & 0 & $u_{1}$ & 0 \\
$e_{4}$ & 0 & $2 e_{4}$ & $-e_{2}$ & 0 & $u_{2}$ & 0 & 0 \\
$u_{1}$ & $-\lambda u_{1}$ & $-u_{1}$ & 0 & $-u_{2}$ & 0 & 0 & 0 \\
$u_{2}$ & $-\lambda u_{2}$ & $u_{2}$ & $-u_{1}$ & 0 & 0 & 0 & 0 \\
$u_{3}$ & $-u_{3}$ & 0 & 0 & 0 & 0 & 0 & 0
\end{tabular},$\lambda=-1 / 2 ;$

- $\overline{\mathfrak{g}}$ is solvable: $5.10 .1(\lambda=\mu=-1), 4.8 .1(\lambda=\mu=-1), 4.9 .1(\lambda=0, \mu=-2)$, 4.11.1 $(\lambda=\mu=-1)$, 4.14.1 ( $\lambda=0, \mu=-2)$, 4.21.1 ( $\mu=-1-\lambda(\lambda \neq-1,-3 / 2))$, 3.8.1 $(\lambda=\mu=-1), 3.13 .1(\lambda=-\mu-1(\mu \neq 0,1 / 2,-1 / 3,-1)), 3.16 .1(\lambda=-2 \mu), 3.20 .1$ $(\lambda=-\mu-1(\lambda \neq 0,1 / 2,-1,-3 / 2)), 3.22 .1(\lambda=-2 \mu(\lambda \neq 0)), 3.23 .1(\lambda=0), 3.27 .1$ ( $\lambda=-1 / 2), 3.29 .1(\mu=-2), 2.2 .1(\lambda=\mu=-1), 2.4 .1(\lambda=0, \mu=-2), 2.9 .1(\lambda=-\mu-1$ $(\lambda \neq 1 / 2,0,-1,-2 / 3,-3 / 2)), 2.16 .1(\lambda=-1 / 2), 2.19 .1(\lambda=-2), 1.2 .1(\lambda=-\mu-1$ $(\mu \neq-1 / 3,-3 / 2,0,-2 / 3)), 1.4 .1(\lambda=-2 \mu(\lambda \neq 0)), 1.7 .1(\lambda=-2)$. The Ricci tensors are zero.

II. Any pair $(\overline{\mathfrak{g}}, \mathfrak{g})$ that admits only trivial affine connection, the curvature and torsion tensors are zero (i.e. if $\overline{\mathfrak{g}}$ is nonsolvable then d.n.m $=9.1 .1,8.1 .1,7.1 .1,7.2 .1,6.2 .1$, 
6.3.1, 6.4.1( $\not \neq 1 / 2), 5.1 .1,4.2 .1(\lambda \neq 1 / 2), 4.3 .1,4.5 .1$, if $\overline{\mathfrak{g}}$ is solvable then d.n.m $=6.5 .1$, 5.4.1, 5.5.1, 5.6.1, 5.7.1, 5.8.1, 5.9.1, 5.10.1 $\left(\lambda^{2}+\mu^{2} \neq 0,(\lambda-1)^{2}+(\mu+1)^{2} \neq 0\right.$, $\left.(\lambda-1 / 2)^{2}+\mu^{2} \neq 0\right), 4.4 .1,4.6 .1,4.7 .1,4.8 .1\left((\lambda+1)^{2}+(\mu-1)^{2} \neq 0, \lambda^{2}+(\mu-1 / 2)^{2} \neq 0\right.$, $\left.\lambda^{2}+\mu^{2} \neq 0\right), 4.9 .1\left(\lambda^{2}+\mu^{2} \neq 0\right), 4.11 .1\left(\lambda^{2}+\mu^{2} \neq 0,(\mu+1)^{2}+(\lambda-1)^{2} \neq 0\right.$, $\left.\mu^{2}+(\lambda-1 / 2)^{2} \neq 0\right)$, 4.12.1, 4.13.1, 4.14.1 $\left((\mu-2)^{2}+\lambda^{2} \neq 0\right), 4.15 .1,4.16 .1,4.17 .1$, 4.18.1, 4.19.1, 4.20.1 $(\lambda \neq 0,-1), 4.21 .1(\mu \neq 0, \mu \neq 1 / 2, \mu \neq 1-\lambda), 4.22 .1,3.1 .1,3.2 .1$, 3.6.1, 3.7.1 $(\lambda \neq 0,1 / 2), 3.8 .1\left(\lambda^{2}+(\mu-1 / 2)^{2} \neq 0, \lambda^{2}+\mu^{2} \neq 0,(\lambda-1 / 2)^{2}+\mu^{2} \neq 0\right.$, $\left.(\lambda+1)^{2}+(\mu-1)^{2} \neq 0,(\lambda-1)^{2}+(\mu+1)^{2} \neq 0\right), 3.9 .1,3.10 .1,3.11 .1,3.12 .1,3.13 .1$ $(\mu \neq 0, \mu \neq 1-\lambda, \mu \neq 1 / 2, \mu \neq \lambda-1, \mu \neq \lambda / 2), 3.16 .1,3.17 .1(\lambda \neq 0), 3.18 .1,3.19 .1$ $(\lambda \neq 0,-1), 3.20 .1(\lambda \neq 0, \lambda \neq 1 / 2, \mu \neq 0, \mu \neq 1 / 2, \mu \neq 1-\lambda), 3.21 .1(\lambda \neq 0), 3.22 .1$ $(\lambda \neq 2 \mu), 3.23 .1(\lambda \neq 2 / 3,1 / 2,3 / 4,3.24 .1,3.26 .1,3.27 .1(\lambda \neq 0,1 / 2), 3.28 .1,3.29 .1$ $(\mu \neq 0,1 / 2), 3.30 .1,3.31 .1,2.1 .1(\lambda \neq 0,1 / 2), 2.2 .1\left((\lambda-1)^{2}+(\mu-1)^{2} \neq 0\right), 2.3 .1,2.4 .1$ $\left(\lambda^{2}+\mu^{2} \neq 0, \lambda^{2}+(\mu-2)^{2} \neq 0\right), 2.5 .1,2.6 .1,2.8 .1(\lambda \neq 0,1 / 2,1,-1), 2.9 .1(\lambda \neq 1 / 2$, $\lambda \neq 0, \lambda \neq 1-\mu, \lambda \neq 2 \mu, \lambda \neq \mu+1, \mu \neq 0, \mu \neq 1 / 2), 2.10 .1,2.11 .1,2.12 .1,2.14 .1,2.16 .1$ $(\lambda \neq 0,1 / 2), 2.19 .1(\lambda \neq 0,1 / 2), 2.21 .1(\lambda \neq 0,1 / 2,2 / 3,3 / 4), 2.22 .1,1.2 .1(\mu \neq \lambda+1$, $\mu \neq 2 \lambda, \mu \neq 1-\lambda, \mu \neq \lambda / 2, \lambda \neq 1 / 2), 1.4 .1(\mu \neq 2 \lambda), 1.7 .1(\lambda \neq 0,2,1 / 2), 1.9 .1$, see [2]), admits the locally equiaffine connection.

Proof. If, for example, $(\overline{\mathfrak{g}}, \mathfrak{g})$ is the space 8.1.1 $(\mathfrak{s l}(3, \mathbb{R})),\left.\Lambda\right|_{\mathfrak{g}}=\lambda, \Lambda$ is $\mathfrak{g}$-invariant $\Rightarrow$ $\Lambda\left(u_{1}\right)=\Lambda\left(u_{2}\right)=\Lambda\left(u_{3}\right)=0$, then the torsion and Ricci tensors are zero, $\operatorname{tr} \Lambda\left(e_{i}\right)=0, i=1,8$, $\left(\Lambda\left(e_{i}\right) \in \mathfrak{s l}(3, \mathbb{R})\right) \Rightarrow$ the connection is equiaffine (and locally equiaffine). In the other cases are similarly.

\subsection{The curvature tensor is not zero for some connections}

Theorem 3.2. I. If the pair $(\overline{\mathfrak{g}}, \mathfrak{g})$ admits only trivial equiaffine connection, the curvature tensor is not zero, the torsion tensor is zero, then $(\overline{\mathfrak{g}}, \mathfrak{g})$ is equivalent to one and only one of the pairs

\begin{tabular}{c|ccccccc}
4.21 .2$. & $e_{1}$ & $e_{2}$ & $e_{3}$ & $e_{4}$ & $u_{1}$ & $u_{2}$ & $u_{3}$ \\
\hline$e_{1}$ & 0 & $(1-\lambda) e_{2}$ & $(3 \lambda-1) / 2 e_{3}$ & $(1+\lambda) / 2 e_{4}$ & $u_{1}$ & $\lambda u_{2}$ & $(1-\lambda) / 2 u_{3}$ \\
$e_{2}$ & $(\lambda-1) e_{2}$ & 0 & $e_{4}$ & 0 & 0 & $u_{1}$ & 0 \\
$e_{3}$ & $(1-3 \lambda) / 2 e_{3}$ & $-e_{4}$ & 0 & 0 & 0 & 0 & $u_{2}$ \\
$e_{4}$ & $-(1+\lambda) / 2 e_{4}$ & 0 & 0 & 0 & 0 & 0 & $u_{1}$ \\
$u_{1}$ & $-u_{1}$ & 0 & 0 & 0 & 0 & 0 & 0 \\
$u_{2}$ & $-\lambda u_{2}$ & $-u_{1}$ & 0 & 0 & 0 & 0 & $e_{4}$ \\
$u_{3}$ & $(\lambda-1) / 2 u_{3}$ & 0 & $-u_{2}$ & $-u_{1}$ & 0 & $-e_{4}$ & 0
\end{tabular}

\begin{tabular}{c|cccccc}
$3.13 .4,-1<\mu<0$ & $e_{1}$ & $e_{2}$ & $e_{3}$ & $u_{1}$ & $u_{2}$ & $u_{3}$ \\
\hline$e_{1}$ & 0 & $(1+\mu) e_{2}$ & $(1-\mu) e_{3}$ & $u_{1}$ & $(1+2 \mu) u_{2}$ & $\mu u_{3}$ \\
$e_{2}$ & $-(\mu+1) e_{2}$ & 0 & 0 & 0 & 0 & $u_{2}$ \\
$e_{3}$ & $(\mu-1) e_{3}$ & 0 & 0 & 0 & 0 & $u_{1}$ \\
$u_{1}$ & $-u_{1}$ & 0 & 0 & 0 & 0 & $e_{2}$ \\
$u_{2}$ & $-(2 \mu+1) u_{2}$ & 0 & 0 & 0 & 0 & 0 \\
$u_{3}$ & $-\mu u_{3}$ & $-u_{2}$ & $-u_{1}$ & $-e_{2}$ & 0 & 0
\end{tabular}




\begin{tabular}{c|cccccc}
$3.20 .5, \mu \geq 1 / 3$ & $e_{1}$ & $e_{2}$ & $e_{3}$ & $u_{1}$ & $u_{2}$ & $u_{3}$ \\
\hline$e_{1}$ & 0 & $2 \mu e_{2}$ & $(1-\mu) e_{3}$ & $u_{1}$ & $(1-2 \mu) u_{2}$ & $\mu u_{3}$ \\
$e_{2}$ & $-2 \mu e_{2}$ & 0 & 0 & 0 & $u_{1}$ & 0 \\
$e_{3}$ & $(\mu-1) e_{3}$ & 0 & 0 & 0 & 0 & $u_{1}$ \\
$u_{1}$ & $-u_{1}$ & 0 & 0 & 0 & 0 & 0 \\
$u_{2}$ & $(2 \mu-1) u_{2}$ & $-u_{1}$ & 0 & 0 & 0 & $e_{3}$ \\
$u_{3}$ & $-\mu u_{3}$ & 0 & $-u_{1}$ & 0 & $-e_{3}$ & 0
\end{tabular}

\begin{tabular}{c|ccccc}
2.9 .3$. & $e_{1}$ & $e_{2}$ & $u_{1}$ & $u_{2}$ & $u_{3}$ \\
\hline$e_{1}$ & 0 & $(1-\mu) e_{2}$ & $u_{1}$ & $(1-2 \mu) u_{2}$ & $\mu u_{3}$ \\
$e_{2}$ & $(\mu-1) e_{2}$ & 0 & 0 & 0 & $u_{1}$ \\
$u_{1}$ & $-u_{1}$ & 0 & 0 & 0 & 0
\end{tabular},$\mu=2$.

In these cases $\overline{\mathfrak{g}}$ is solvable.

II. If the pair $(\overline{\mathfrak{g}}, \mathfrak{g})$, admitting only trivial affine connection (with nonzero curvature tensor and zero torsion tensor), does not admit locally equiaffine connection, then $(\overline{\mathfrak{g}}, \mathfrak{g})$ is equivalent to one of the pairs:

\begin{tabular}{cc|cccccc} 
& 2.9 .12$. & $e_{1}$ & $e_{2}$ & $u_{1}$ & $u_{2}$ & $u_{3}$ & \\
\cline { 2 - 6 } & $e_{1}$ & 0 & $-e_{2}$ & $u_{1}$ & $-2 u_{2}$ & $2 u_{3}$ & \\
& $e_{2}$ & $e_{2}$ & 0 & 0 & 0 & $u_{1}$ & \\
& $u_{1}$ & $-u_{1}$ & 0 & 0 & $e_{2}$ & 0 & \\
& $u_{2}$ & $2 u_{2}$ & 0 & $-e_{2}$ & 0 & $-e_{1}$ & \\
3.8 .8$. & $u_{3}$ & $-2 u_{3}$ & $-u_{1}$ & 0 & $e_{1}$ & 0 & \\
\hline$e_{1}$ & $e_{1}$ & $e_{2}$ & $e_{3}$ & $u_{1}$ & $u_{2}$ & $u_{3}$ \\
$e_{2}$ & 0 & 0 & $e_{3}$ & $u_{1}$ & 0 & 0 \\
$e_{3}$ & $-e_{3}$ & $-e_{3}$ & 0 & 0 & 0 & $u_{3}$ \\
$u_{1}$ & $-u_{1}$ & 0 & 0 & 0 & $e_{3}$ & 0 \\
$u_{2}$ & 0 & $-u_{2}$ & 0 & $-e_{3}$ & 0 & $2 e_{2}-e_{1}$ \\
$u_{3}$ & 0 & $u_{3}$ & $-u_{1}$ & 0 & $e_{1}-2 e_{2}$ & 0
\end{tabular}

III. Any pair $(\overline{\mathfrak{g}}, \mathfrak{g})$ that admits only trivial affine connection, the curvature tensor is not zero, the torsion tensor is zero, except 2.9.12 and 3.8.8 (i.e. if $\overline{\mathfrak{g}}$ is nonsolvable then d.n. $m=4.11 .2,4.13 .2,4.13 .3,2.1 .2,2.3 .2,2.3 .3$, if $\overline{\mathfrak{g}}$ is solvable then d.n. $m=5.10 .2$, 4.8.10, 4.11.4, 4.20.2, 4.21.2 $(\lambda \neq 1), 3.8 .9,3.13 .2(\mu \neq 0,1 / 2,1 / 4), 3.13 .4,3.14 .2$, 3.19.16, 3.20.4 $(\lambda \neq 0,1 / 4), 3.20 .5(\mu \neq 1 / 2), 3.23 .2,3.27 .2,2.8 .6,2.9 .3(\mu \neq$ $0,1 / 2,1 / 4), 2.16 .2$, see [2]) admits the locally equiaffine connection.

The Ricci tensors has the form (in the other cases Ricci tensors are zero):

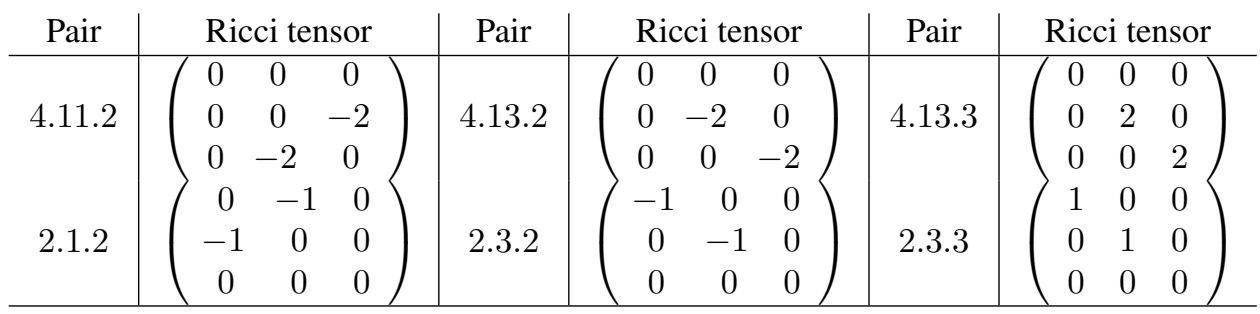


Proof. In the case 2.9.12 we have $\Lambda$ is $\mathfrak{g}$-invariant $\Rightarrow \Lambda\left(u_{1}\right)=\Lambda\left(u_{2}\right)=\Lambda\left(u_{3}\right)=0$, Ricci tensor has the form

$$
\left(\begin{array}{ccc}
0 & 0 & 0 \\
0 & 0 & -3 \\
0 & -2 & 0
\end{array}\right)
$$

and Ricci tensor is not symmetric. Also we have $\operatorname{tr} \Lambda\left(e_{1}\right) \neq 0 \Rightarrow$ the connection is not locally equiaffine (and equiaffine too). In the case 3.8.8 Ricci tensor as in the case 2.9.12, also we have $\operatorname{tr} \Lambda\left(e_{1}-2 e_{2}\right) \neq 0 \Rightarrow$ the connection is not locally equiaffine (and equiaffine too). In other cases the connection is locally equiaffine $(\operatorname{tr} \Lambda([x, y])=0$ for all $x, y \in \overline{\mathfrak{g}})$, the Ricci tensors have the form, presented in the theorem, and Ricci tensors are symmetric.

So for all three-dimensional homogeneous spaces, not admitting connections with nonzero torsion tensor, it is determined under what conditions the connection is equiaffine (locally equiaffine). In addition, equiaffine (locally equiaffine) connections and Ricci tensors are written out in explicit form. For example, there are only two spaces (not admitting connections with nonzero torsion tensor) that admit affine connections, but do not admit locally equiaffine connections. There are no pairs, admitting nontrivial equiaffine connections with nonzero curvature tensor and only zero torsion tensor. In this work we use the algebraic approach for description of connections, methods of the theory of Lie groups, Lie algebras and homogeneous spaces. The results can find applications in mathematics and physics, since many fundamental problems in these fields are reduced to the study of invariant objects on homogeneous spaces.

\section{ORCID iDs}

Natalya P. Mozhey (D) https://orcid.org/0000-0001-9237-7208

\section{References}

[1] S. Kobayashi, Transformation groups in differential geometry, Classics in Mathematics, Springer-Verlag, Berlin, 1995, doi:10.1007/978-3-642-61981-6, reprint of the 1972 edition.

[2] N. P. Mozhey, Torsion free affine connections on three-dimensional homogeneous spaces, Sib. Èlektron. Mat. Izv. 14 (2017), 280-295, doi:10.17377/semi.2017.14.026.

[3] K. Nomizu, Invariant affine connections on homogeneous spaces, Amer. J. Math. 76 (1954), 33-65, doi:10.2307/2372398.

[4] K. Nomizu and T. Sasaki, Affine differential geometry, volume 111 of Cambridge Tracts in Mathematics, Cambridge University Press, Cambridge, 1994, geometry of affine immersions. 